\title{
Zum historischen Wandel übersetzungswissenschaftlicher Termini unter dem Blickwinkel der Dekonstruktion
}

\author{
Emra Büyüknisan (D), Mersin \\ https://doi.org/10.37583/diyalog.1030844
}

\begin{abstract}
Deutsch)
Der Ansatz der Dekonstruktion von Jacques Derrida (1967) stellt sich gegen die Universalität sprachlicher Bedeutungskonstrukte, während sie Wörtern einen historischen Charakter zuordnet und auf die Relevanz von Subjekt, Zeit und Ort, insbesondere im Rahmen der schriftlichen Kommunikation hinweist. Die Terminologieforschung jedoch fordert eine möglichst genaue konzeptionelle Definition von Termini, damit Fachkommunikation tunlichst eindeutig verlaufen kann. In der Übersetzungswissenschaft hingegen besteht insbesondere im Rahmen theoretischer Forschungsarbeiten neben der Anforderung an eine standardisierte Terminusdefinition auch die Kritik an der Varietät der Definitionen eines Terminus. Die vorliegende Arbeit fokussiert sich auf die Darstellung einer neuen Perspektive für die Herangehensweise an Definitionen übersetzungswissenschaftlicher Terminologie. Mit Bezug auf Paradigmenwechsel innerhalb der Übersetzungswissenschaft wird in diesem Zusammenhang die historische Entwicklung der konzeptionellen Inhalte der Termini Äquivalenz, Übersetzung und Übersetzer(in) unter dem theoretischen Blickwinkel der Dekonstruktion betrachtet. Diese Arbeit soll dabei den Beleg dafür erbringen, dass Definitionen bestimmter Termini der Übersetzungswissenschaft nicht als konkret begrenzt und universal aufgefasst werden sollten.
\end{abstract}

Schlüsselwörter: Übersetzungswissenschaft, Dekonstruktion, Paradigma, Terminologie, Äquivalenz, Translation, Translator.

\section{Abstract (English) \\ On the Historical Development of the Terms of Translation Studies From the Perspective of Deconstruction Theory}

The theory of deconstruction of Jacques Derrida stands against the universality of lingual meaning structures and points to the relevance of time, place and subject especially in the context of written communication. Terminology researches, however, claim that the conceptual definition of terms must be as precise as possible so that technical communication can proceed as clearly as possible. In translation studies, on the other hand, in the context of theoretical research, besides the requirement for a standardized definition of terms, there is also criticism about the variety of the definitions of one and the same term. This work aims to present a new perspective to the approach of definitions of terminology in translation studies. In this context, the historical development of the conceptual content of the terms equivalence, translation and translator is investigated on the basis of the theoretical perspective of deconstruction in relation to paradigm changes within translation studies.

Keywords: Translation Studies, Deconstruction, Paradigm, Terminology, Equivalence, Translation, Translator.

Einsendedatum: 17.08.2021

Freigabe zur Veröffentlichung: 01.12.2021 


\section{EXTENDED ABSTRACT}

Basic properties of language include the function of designation and expression. Without these functions, many processes linked to communication would largely fail, and nothing could be spoken of research and science. In contrast to other language categories, academic language requires precise definitions that are generally recognized in the subject area, particularly regarding terminology. Translation studies use terms to communicate as well, which is why the same requirement applies here.

However, translation approaches are criticized in the context of the appropriate specialist literature both regarding their theoretical inadequacy and their lack of reference to one another, as well as their terminological or conceptual diversity and even incoherence. In order to better understand the reasons for partial theories and terminological diversity within translation studies and to be able to interpret these theories more accurately, the project to combine the theory of deconstruction by Jacques Derrida (1967) with the historical development of translation research seems to be fundamental.

Also the fact that reflexive modern sciences have severely excluded variable factors such as subject, time and place from theoretical research - though these include dimensions and realities of the world and of human perception - , and that modern scientists appreciate exclusively universalistic, generally valid, verifiable and objective statements today leads to the necessity to take up these variable factors again, because without them facts of the world are only partially or even not comprehensible and definable. Indeed, the weight of history can help counterbalance many current debates within translation studies. Another aim of this paper is to redirect the focus within translation research to variable factors such as subject, time and place and, in particular, to reinterpret the variety of term definitions in the frame of translation research from this point of view.

Another motivation for this research consists of the hypothesis that the expectation of concepts that are clearly delimited in terms of content can be misleading within the frame of translation research. In this context, this work provides evidence that definitions of certain terms in translation research cannot be accepted unreservedly as concretely limited and universally valid.

Because of these arguments, the three terms equivalence, translation, and translator are examined based on of their definitions within the field of translation research. These terms are probably among the most frequently used and processed terms in the theoretical subject areas of translation. They can already be found in the early period of translation researches up to more current approaches.

In this study, it is aimed to explain the variety of definitions of these terms using the theory of deconstruction. The starting point here is the hypothesis that deconstruction can broaden the horizons in translation research because it assigns words a historical character. In particular, terms have not been interpreted from a historical perspective in this field yet. This leads to the assumption that the conception of a theoretical or conceptual "confusion" (Unübersichtlichkeit, Stolze 2011, 9-12) in the field perhaps arose from precisely this attitude.

According to Jacques Derrida and Peter Václav Zima, oral communication is unique and specific, while written communication connected with the concept of writing must be repeatable and citable. This notion is called iterability or infinite substitutions in the deconstruction approach. What is meant here is not a simple repetition, but rather, combined with the variability of the recipient, a repetition in the sense of otherness and renewal. 
This work is not about the subjective understanding and interpretation of terms, but about the variety of their definition contents in the course of historical developments. The terms of iterability and dissemination are therefore not dealt with on a subjective or individual level. In this study, differences in the interpretation of terms are explained with different perspectives of research societies from different historical epochs. In this context, the historical development of the conceptual content of the terms equivalence, translation and translator is investigated on the basis of the theoretical perspective of deconstruction in relation to paradigm changes within translation studies. 


\section{Einführung}

\section{Problemdarstellung, Ziel und theoretischer Hintergrund}

Grundlegende Eigenschaften der Sprache sind u.a. die Bezeichnungs- und die Ausdrucksfunktion (vgl. Bühler 1965: 28). Ohne diese Funktionen der Sprache würden viele an Kommunikation gebundene Vorgänge größtenteils scheitern und es könnte auch nicht von Forschung und Wissenschaft gesprochen werden (siehe auch Derrida 1974: 1214). Im Gegensatz zu anderen Sprachkategorien fordert die Wissenschaftssprache präzise und im Fachbereich allgemein anerkannte Definitionen besonders in Bezug auf Termini. Auch die Übersetzungswissenschaft verständigt sich mit Hilfe von Termini, weshalb die gleiche Forderung nicht zuletzt auch hier gültig ist.

Übersetzungswissenschaftliche Ansätze werden jedoch im Rahmen der Fachliteratur sowohl hinsichtlich ihrer theoretischen Mangelhaftigkeit und ihrer Bezugslosigkeit zueinander, als auch hinsichtlich ihrer terminologischen bzw. begrifflichen Vielfalt und sogar Inkohärenz kritisiert. ${ }^{1}$ Um die Gründe für Teiltheorien und terminologische Vielfalt innerhalb der Übersetzungswissenschaft besser verstehen zu können und um diese Theorien an sich zutreffender interpretieren zu können, scheint das Vorhaben, die Theorie der Dekonstruktion von Jacques Derrida (1967) mit der historischen Entwicklung der übersetzungswissenschaftlichen Forschung zu verbinden, von grundlegender Bedeutung zu sein.

Auch die Tatsache, dass reflexive moderne Wissenschaften variable Faktoren wie Subjekt, Zeit und Ort aus theoretischen Forschungen schwerwiegend ausgeschlossen haben, obwohl diese u.a. Dimensionen und Realitäten der Welt und der menschlichen Auffassung sind, und dass Wissenschaftler(innen) der Moderne ausschließlich universalistische, allgemeingültige, verifizierbare und objektive Aussagen schätzen (vgl. Stachowitz 1973; Derrida 1974: 110; Wengenroth 2012: 12f), führt heute zu der Notwendigkeit, diese variablen Faktoren wiederaufzunehmen, denn ohne diese sind Fakten der Welt nur teilweise oder nicht begreifbar und definierbar (vgl. Hörz 1999: 7; Derrida 1972: 431). Im Lichte historischer und vielleicht sogar subjektiver Entwicklungen können übersetzungswissenschaftliche Theorien neu interpretiert werden. Die Berücksichtigung der Geschichte kann nämlich dazu beitragen, viele gegenwärtige Debatten innerhalb der Übersetzungswissenschaft auszugleichen (vgl. Pym 2015: 105). Eine weitere Absicht dieser Arbeit besteht also darin, innerhalb der Übersetzungswissenschaft das Augenmerk erneut auf variable Faktoren wie Subjekt, Zeit und Ort $\mathrm{zu}$ richten und insbesondere die Vielfältigkeit von übersetzungswissenschaftlichen Terminusdefinitionen aus diesem Blickwinkel neu zu interpretieren.

Ein weiterer Beweggrund für diese Forschung besteht aus der Hypothese, dass die Erwartung auf inhaltlich konkret abgegrenzte Konzepte im Rahmen der Übersetzungswissenschaft irreführend sein kann. In diesem Zusammenhang soll diese

\footnotetext{
${ }^{1}$ S. bspw. Wilss 1977: Kap. III; Snell-Hornby 1988: 1-5; Koller 1995: 191f; Toury 1995: 2f; Schäffner 2000: 205; Gile 2009: 1-4; Stolze 2011: 9-12; 94; Pym 2014: 1-3.
} 
Arbeit den Beleg dafür erbringen, dass Definitionen bestimmter Termini der Übersetzungswissenschaft nicht uneingeschränkt als konkret begrenzt und universal gültig akzeptiert werden können.

Auf der Basis dieser Argumente werden in dieser Arbeit die drei Termini Äquivalenz, Übersetzung und Übersetzer(in) in Hinsicht auf ihre Definitionen innerhalb des Fachbereichs der Übersetzungswissenschaft untersucht. Diese Begriffe gehören wohl zu den am häufigsten verwendeten und bearbeiteten Termini in den theoretischen Ansätzen des betreffenden Fachbereichs.und sind bereits in den ersten übersetzungswissenschaftlichen Forschungsarbeiten bis hin zu aktuelleren Ansätzen vorzufinden. $^{2}$

Nach der Darstellung der Problematik und des entsprechenden Forschungsstands ergibt sich die Frage, in welcher Hinsicht die Vielfältigkeit der Definitionen von übersetzungswissenschaftlichen Termini argumentiert werden kann. Dies ist ein Versuch, die genannte Vielfältigkeit anhand der Theorie der Dekonstruktion zu erläutern. Ihren Ausgangspunkt nimmt diese Untersuchung hierbei in der Hypothese, dass die Dekonstruktion den Horizont in der übersetzungswissenschaftlichen Debatten erweitern kann (vgl. u.a. Zima 1994: 88), da sie Wörtern einen historischen Charakter zuweist (vgl. Derrida 1974: 29). Insbesondere fachinterne Termini wurden bisher in der Fachliteratur überwiegend nicht aus historischer Perspektive interpretiert. Daraus erwächst die Annahme, dass die Auffassung einer theoretischen oder begrifflichen „Unübersichtlichkeit“ (Stolze 2011: 9-12) im Forschungsfeld vielleicht genau aus dieser Haltung entstanden ist.

Nach Jacques Derrida und Peter Václav Zima ist mündliche Kommunikation einmalig und bestimmt, während schriftliche Kommunikation verbunden mit dem Schriftbegriff wiederholbar und zitierbar sein muss. Diese Vorstellung wird im Ansatz der Dekonstruktion als Iterabilität oder unendliche Substitutionen bezeichnet (vgl. Derrida 1974: 16, 24, 33; Derrida 1972: 477; Zima 1994: 55). Hier ist keine einfache Wiederholung, sondern, verbunden mit der Variabilität des Empfängers, eine Wiederholung im Sinne von Andersheit und Erneuerung gemeint.

In dieser Arbeit geht es nicht um das subjektive Verstehen und Interpretieren von Termini, sondern um die Vielfalt ihrer Definitionsinhalte im Zuge historischer Entwicklungen. Die Begriffe der Iterabilität und der Dissemination werden hier deshalb nicht auf subjektiver oder individueller Ebene bearbeitet. In dieser Untersuchung sollen die Interpretationsdifferenzen der genannten exemplarischen Termini anhand der unterschiedlichen Denkperspektiven von Forschungsgesellschaften unterschiedlicher historischer Epochen erklärt werden. Da es sich u.a. um Termini und ihre Definitionen, darüber hinaus um Paradigmenwechsel in der Übersetzungswissenschaft handelt,

\footnotetext{
2 Der Begriff Äquivalenz wurde bspw. von Nida (1964), Kade (1968), Nida / Taber (1969), Catford (1965), Schreiber (1993), Koller (1979, 1992, 2004), Snell-Hornby (1986), Newmark $(1988,1991)$ und House (1997), der Begriff Übersetzen u.a. von Reiß (1971, 1976), Koller (1992), Gerzymisch-Arbogast (1994), Searle (1976), Hönig / Kußmaul (1982) und der Begriff Übersetzer u.a. von Holz-Mänttäri $(1984,1986)$, Nord (1989, 1991), von Flotow (1997), Tymoczko (2000), Gouanvic $(1999,2005)$ und Prunč $(2004,2007)$ intensiv bearbeitet, um nur einige von vielen Ansätzen genannt zu haben (s. Stolze 2011).
} 
erscheint es sinnvoll, zunächst auf die Konzepte Terminus und Paradigma kurz einzugehen, bevor der historische Wandel der übersetzungswissenschaftlichen Termini unter dem Blickwinkel der Dekonstruktion behandelt wird.

\section{Der Terminus}

Der Begriff Terminus stammt von dem griechischen Wort terma, das ,Ende, Ziel“ bedeutet, und dem lateinischen Wort terminus ab, das in Form von, inhaltlich abgegrenzter, festumrissener Begriff, festgelegter (Grenz-)punkt ${ }^{\star}$ definiert wird (Kluge 2002; Wahrig 1997: 1220). Heute wird dieses Sprachzeichen in Wörterbüchern dem Begriffsinhalt von ,Grenze, Ende, Ziel` zugeordnet. Das Deutsche Institut für Normung definiert den Begriff Terminus wie folgt: ,Das zusammengehörige Paar aus einem Begriff und seiner Benennung als Element einer Terminologie“ (DIN 2342) (zitiert nach Arntz / Picht 2004: 37).

Historisch betrachtet entwickelten sich Termini besonders in der Zeit nach der industriellen Revolution, im späten 19. und 20. Jahrhundert, als Forschung und Technologie in den Vordergrund traten (vgl. Sonneveld / Loening 1993). Nachdem das Bedürfnis nach definitiv eng eingegrenzten Wörtern für die Bezeichnung und eine präzise Beschreibung von z.B. unterschiedlichen Fachgegenständen, -prozessen oder -konzepten stieg, entwickelten sich bis heute Terminologien in unterschiedlichsten Fachbereichen. ${ }^{3}$ Terminologie ist der Gesamtbestand der Begriffe und ihrer Benennungen in einem Fachgebiet (DIN 2342: 16). Das Aufkommen von Fachsprachen und Fachkommunikation gehört selbstverständlich auch $\mathrm{zu}$ dieser Entwicklung. Fachkommunikation ist ,[...] die von außen oder von innen motivierte bzw. stimulierte, auf fachliche Ereignisse oder Ereignisabfolgen gerichtete Exteriorisierung und Interiorisierung von Kenntnissystemen und kognitiven Prozessen“ (Hoffmann 1993: 614).

Für die Intention, den historischen Wandel exemplarisch dargestellten übersetzungswissenschaftlichen Termini darzustellen, ist zunächst also festzuhalten, dass ein Terminus eine einem begrifflichen Inhalt zugeordnete Bezeichnung ist und dass der genannte begriffliche Inhalt dieser Bezeichnung möglichst genau bestimmt und eingegrenzt sein sollte.

Wie kann demnach die begriffliche Vielfalt innerhalb der Übersetzungswissenschaft erklärt werden? In diesem Zusammenhang soll zunächst auf den Begriff Paradigma näher eingegangen werden, der anschließend mit der Dekonstruktion von Derrida relativiert werden soll.

\footnotetext{
${ }^{3}$ Demnach wäre die Bewertung von Wörtern, die ungefähr vor dem späten 19. Jahrhundert benutzt wurden, in der Kategorie von Termini irreführend.
} 


\section{Das wissenschaftliche Paradigma}

Da es in dieser Arbeit um den historischen Wandel von Terminidefinitionen geht, ist ein Exkurs auf den Begriff Paradigma grundlegend. Das vom Griechischen paradeigma (Beispiel) abstammende und im Wahrig (1997, 940) mit der Definition als ,Musterbeispiel' (Wahrig: 1997, 940) erklärte Wort ist im Zusammenhang von Wissenschaften der Ausdruck für „das, was den Mitgliedern einer wissenschaftlichen Gemeinschaft gemeinsam ist, und umgekehrt besteht eine wissenschaftliche Gemeinschaft aus Menschen, die ein Paradigma teilen“ (Kuhn 2003: 187). In der wissenschaftlichen Auseinandersetzung ist unter einem Paradigma eine Art Denkmuster in Bezug auf grundlegende Probleme und Methoden eines Fachbereiches zu verstehen, das u.a. auch als ,Supertheorie" bezeichnet wird (vgl. Bleicher 1992: 42). Ein wissenschaftliches Paradigma ist zugleich die Gesamtheit von Theorien, philosophischen Grundlagen, Wertvorstellungen und Musterkonzepten, die das Forschungsfeld und die Vorgehensweise einer wissenschaftlichen Gemeinschaft bestimmen.

„Paradigmen entstehen, entwickeln und verändern sich nicht im luftleeren Raum, sondern in ganz bestimmten wissenschaftlich-institutionellen, politischen, kulturellen und sozioökonomischen Zusammenhängen“ (Koller 2000: 121). Abhängig von historischen, kulturellen, geographischen oder politischen Ereignissen bzw. Veränderungen kann es im Laufe der Zeit immer wieder zu Paradigmenwechseln in den Wissenschaften kommen, auch wenn diese Verbindung zwischen historischer Entwicklung und wissenschaftlichem Werdegang bisher nicht immer offen dargestellt wurde. Obwohl sie sich das objektive Arbeiten zum Ziel gemacht haben, können sogar Wissenschaftler(innen) als Subjekte unter dem Einfluss ihres geschichtlichen Kontextes stehen. Dies wird im nächsten Kapitel dieser Arbeit verdeutlicht.

Während die Gültigkeit bestimmter Paradigmen innerhalb einer Wissenschaftsdisziplin länger anhält, können andere ihre Stellung schneller neuen Paradigmen überlassen, wie es auch Kuhn folgendermaßen formuliert: „Paradigmen bleiben jedoch - wenn auch für längere Zeit - nur für eine bestimmte Zeitspanne gültig und überlassen dann ihren Platz einem neuen Paradigma“ (Kuhn 2003: Abschnitt 10).

Auch diese den wissenschaftlichen Paradigmenwechsel betreffende Definition weiterführend wird im Anschluss der Ansatz der Dekonstruktion von Derrida, der später von Zima (1994) literaturwissenschaftlich interpretiert wird, mit der Problematik der begrifflichen Definitionsvielfalt der Übersetzungswissenschaft verbunden und interpretiert.

\section{Die Dekonstruktion}

Die Dekonstruktion von Jacques Derrida (1974) ist eine umfassende Philosophie bzw. Methodologiekritik, deren Einzelheiten hier nicht einzeln diskutiert werden sollen. Entscheidend für die Fragestellung dieser Untersuchung ist vielmehr die Neuinterpretation der Dekonstruktion von Zima (1994), die den Ansatz -anders als die Originalintention- aus literaturwissenschaftlicher Perspektive deutet (vgl. Zima 1994: 34) 
und mit seinem Kapitel Babel oder die unmögliche Übersetzung: von Benjamin zu Derrida und de Man auch auf die übersetzungswissenschaftliche Forschungsebene trägt (auch Atkins 1983: 16).

Zunächst ist $\mathrm{zu}$ unterscheiden, dass die Dekonstruktion sich gegen den Logozentrismus von Ferdinand de Saussure stellt (vgl. Zima 1994: 51,82; Derrida 1974: 23). Die Dekonstruktion ,„[...] ist ein radikaler Zweifel am Strukturbegriff (s. Kap. II) [...]“ (ebd.: 31) und lehnt universalistische Theorien in Bezug auf das Schriftzeichen ab. Nach der Dekonstruktion ist die phonetische Schrift, d.h. die mündliche Kommunikation, einmalig, klar und bestimmt. Bei dieser Art von Kommunikation gibt es mindestens einen bestimmten Sender, mindestens einen bestimmten Empfänger und einen bestimmten Ort sowie eine bestimmte Zeit. Die schriftliche Kommunikation dagegen ist zeitlich und räumlich nicht begrenzt. Bei ihr gibt es nur mindestens einen bestimmten Sender, wobei die Bestimmtheit von Empfänger, Zeit und Ort dekonstruiert ist (vgl. Derrida 1974: 23f).

Wörter haben im Rahmen dieser Theorie einen geschichtlichen Hintergrund und können nicht nur als Zeichen bzw. Strukturen der Präsenz aufgefasst werden (vgl. Derrida 1972: 422-442; de Man 1979: 69). Denn nach diesem Ansatz kann das Wort nicht von der Handlung isoliert behandelt werden (vgl. de Man 1979: 49).

Nach Derrida muss das Variable, das Kulturelle, das Historische wieder in die Natur bzw. in die Wissenschaften integriert werden (vgl. Derrida 1972: 431). Derrida entwickelt seine teilweise auf Lévi-Strauss (1968) gegründeten Gedanken so weit fort, dass er zu der Ansicht gelangt, man könne nichts Allgemeingültiges aussagen: „Man muß hier also auf den wissenschaftlichen oder philosophischen Diskurs, auf die episteme verzichten, die die absolute Forderung stellt, zur Quelle, zum Zentrum, zum Grund, zum Prinzip usw. zurückzugehen“ (Derrida 1972: 433). Derrida bringt zum Ausdruck, dass umfassende Einheitlichkeiten innerhalb der Wissenschaften mit der Realität nicht übereinstimmen können, dass die Suche danach nicht realistisch ist, und dass diese Vereinheitlichungsversuche nur synthetischen Charakter aufweisen können (vgl. ebd.). Diese Untersuchung beabsichtigt nicht, Derridas zuletzt genannten Gedanken als theoretischen Rahmen zu verwenden, sondern möchte darauf hinweisen, dass es eine Notwendigkeit ist, in wissenschaftlichen Forschungsarbeiten auch die Historizität und die Variabilität mit einzubauen.

Nach der Dekonstruktion von Derrida ist jede Rezeption von Schriftzeichen eine „,neue Belebung des Originals“ (Derrida 2007: 211; Zima 1994: 88). In diesem Zusammenhang wird der Begriff ,dissémination “ hervorgehoben, der die Streuung der Bedeutungen eines selben Schriftzeichens in unterschiedlichen Kontexten ausdrückt (Zima 1994: 66f).

\section{Struktur vs. Geschichte: Begriffe im historischen Wandel}

An dieser Stelle sei noch einmal zu erwähnen, dass in dieser Arbeit die unterschiedliche Rezeption der Schriftzeichen nicht im Sinne von Rezeptionsunterschieden zwischen einzelnen Subjekten aufgenommen wird. Die Variabilität der Rezeption von 
Schriftzeichen wird hier in Bezug zu den Forschungskreisen aus unterschiedlichen historischen Perioden betrachtet. Es handelt sich hier also um die unterschiedliche Auffassung von Termini im Rahmen bestimmter historischer Perioden, verbunden mit dem derzeitigen historischen Kontext und dem dazugehörigen Paradigma.

Demnach kann im Weiteren gefolgert werden, dass auch übersetzungswissenschaftliche Termini aus ihrem geschichtlichen Hintergrund verstanden und interpretiert werden können bzw. sollten. Dabei kann das Prinzip der Eindeutigkeit in der Definition, das von Seiten der Terminologieforschung eingehalten werden soll, durchaus gültig bleiben, während auch Paradigmenwechsel verbunden mit historischen oder wissenschaftlichen Entwicklungen innerhalb einer Wissenschaft, hier der Übersetzungswissenschaft, eine Rolle spielen können. Im Rahmen der Übersetzungswissenschaft ist zu beobachten, dass die definitorische Vielfalt bestimmter Termini, aus chronologischer Perspektive betrachtet, an Sinn gewinnt.

Grundlegend wird hier erstens auf die Iterabilität und die Dissemination der Dekonstruktion und zweitens auf den Paradigmenwechsel innerhalb der Wissenschaften aufgebaut, so dass auch Termini der Übersetzungswissenschaft von historischen Kontexten abhängige Definitionsänderungen aufweisen können.

Aus diesem Blickwinkel seien nun die drei Begriffe Äquivalenz, Übersetzung und Übersetzer(in) im nächsten Abschnitt umfassender zu betrachten.

\section{Übersetzungswissenschaftliche Begriffe im historischen Wandel}

Auch übersetzungswissenschaftliche Termini können demgemäß aus der Perspektive der Dekonstruktion analysiert werden. Zwar fordert die Terminologieforschung möglichst genau definierte Begriffsinhalte, jede Definition aber kann - so allgemeingültig sie auch zu sein scheint- ihre Gültigkeit nur für eine -längere oder kürzere- bestimmte Zeit bewahren.

Wie in anderen Forschungsdisziplinen, sind im Rahmen der Entwicklungsgeschichte der Übersetzungswissenschaft Paradigmenwechsel zu beobachten. Die Zeitspannen zwischen diesen Paradigmenwechsel können grob in folgende drei historische Phasen eingeteilt werden.

\section{Präskriptive paradigmatische Phase}

Der ersten historischen Phase der Übersetzungsforschungen, die ungefähr Mitte des 20. Jahrhunderts in der Moderne einsetzt, liegt der russische Formalismus des frühen 20. Jahrhunderts $\mathrm{zu}$ Grunde. Obwohl in dieser Zeit noch keine bedeutenden Übersetzungstheorien entwickelt werden, gründen viele Übersetzungstheorien des folgenden Jahrhunderts auf diesen Formalismus. Erstmals versuchen russische Formalisten in den ersten Jahrzehnten des 20. Jahrhunderts die wissenschaftliche Deskription von Kulturprodukten und -systemen in der Literatur zu erstellen. Die Leitidee dieser Strömung ist die Überzeugung, dass die Wissenschaft auch Kultur erfassen muss. 
Tatsächlich bringt die Nachwuchsgeneration der Prager Strömung es so weit, dass in den 60er und 70er Jahren zum Beispiel Jiří Levý, František Miko und Anton Popovič versuchen, die strukturellen Prinzipien, die der literarischen Übersetzung zugrunde liegen, zu beschreiben. Popovič macht mit seinem Ansatz darauf aufmerksam, dass man sich bei der Übersetzungsforschung auf die Unterschiede (shifts) zwischen Original- und Übersetzungstexten fokussieren sollte, anstatt auf Äquivalenz, da Übersetzungen in gewissem Maße Texte verändern (vgl. Pym 2010: 1-7).

Das Prager Interesse an literarischer Translation ist durchaus kein Zufall. Die tschechische Literatur war im Vergleich zur russischen Literatur ein kleineres System und stand unter größerem Einfluss literarischer Übersetzungen. In diesem Zusammenhang publiziert Jan Mukařovský (1936) seine These darüber, dass mit Hilfe von Translation auch Änderungen an nationaler Literatur vorgenommen werden können.

Der Prager linguistische Kreis, zu dem u.a. Roman Jakobson zählt, kommt bereits 1926 zusammen. Jakobsons Flucht aus dem von Deutschland besetzten Prag bringt ihn nach Kopenhagen, Stockholm, New York und Harvard, wo er akademisches Interesse weckt und dadurch einen fundamentalen Einfluss auf die Translationswissenschaft bewirkt. Auch Nikolai Trubetzkoi und Henry Becker stammen aus der Prager Schule. Trubetzkoi vertritt einen Lehrstuhl in Wien, während Becker in Leipzig lebt. Wie aus diesen Entwicklungen zu folgern ist, öffnet sich der Prager Kreis definitiv jenseits den Grenzen von Prag (vgl. ebd.). Durch den Übergang von Akademikern der Prager Schule nach Ostdeutschland und seinen nächsten Generationen wird Jahrzehnte später die Leipziger Schule an der Karl-Marx-Universität gegründet, wodurch die Übersetzungsforschungen sich somit nach Deutschland verlagern und noch verstärkter in Europa wahrgenommen werden.

Unter dem Einfluss des sozialistischen Systems und im Einklang mit den damals vorherrschenden marxistischen Wissenschaftstheorien wird an der Karl-MarxUniversität vom 14. bis zum 17. September 1970 die II. Internationale Konferenz mit dem Titel Grundfragen der Übersetzungswissenschaft veranstaltet. Auf dieser Konferenz wird von der ,[...] Schaffung einer marxistisch-leninistischen Übersetzungstheorie [...] als Ziel der Konferenz und von „sprachmittlerisches System“ als „Übersetzer(in)“ (Maschine) gesprochen (Koller 2000: 127f). Gert Jäger definiert auf derselben Konferenz „den gesellschaftlichen Auftrag der Übersetzungswissenschaft“ als „,einen maximalen Auftrag im Dienste des sozialistischen Systems“ (ebd.).

Otto Kade übt in seiner Konferenzrede Kritik an den USA und ihrer kapitalistischen Wirtschaftsordnung aus. Er behauptet, dass die Idee von der maschinellen Übersetzung in den USA gescheitert sei, da man dort nur nach kapitalistischen Prinzipien handeln würde. Demzufolge wäre der/ die Humanübersetzer(in) preisgünstiger als die maschinelle Übersetzung. Seines Erachtens sei die Verwirklichung der Idee von der maschinellen Übersetzung die Aufgabe des sozialistischen Ostens, da diese nicht kapitalistisch, sondern idealistisch und wissenschaftlich sei. Im Zusammenhang dieser Rede von Kade wird „die Einheit der kommunistischen Weltbewegung“ mit der „Einheit von Theorie und Praxis" verbunden (ebd.: 131f). Wie bekannt hat die Leipziger Schule 
einen wichtigen Stellenwert im Rahmen der Entwicklungsgeschichte der Übersetzungswissenschaft und ist trotz dieser aus wissenschaftlicher Hinsicht nicht sehr weiterbringenden sozialistischen Betonungen keineswegs gering zu schätzen (vgl. Pym 2014: 6).

Diese historische Epoche der Übersetzungswissenschaft fängt mit frühen Übersetzungsforschungen als Teilgebiet der kontrastiven Linguistik mit dem Ziel an, das maschinelle Übersetzen voranzutreiben, und reicht bis ins späte 20. Jahrhundert. Das herrschende Paradigma gründet neben den Einflüssen des russischen Formalismus ${ }^{4}$ auf die universalistische Sprachphilosophie und den Strukturalismus von Ferdinand de Saussure (1916). Das Ziel jeder Übersetzungstheorie wird als die Verwirklichung der maschinellen Übersetzung formuliert und die Ansätze aus dieser Zeit sehen die Zuordnung von Sprachzeichen und -strukturen der einen Sprache mit Sprachzeichen und -strukturen der anderen Sprache vor. In dieser Periode der Übersetzungsforschungen werden neben jenen Zuordnungen auch Formulierungen von Übersetzungsregeln als Algorithmus für die Programmierung von ersten Computern zum Ziel jeder Übersetzungstheorie formuliert (vgl. Stolze 2011: 49).

Der Ansatz der Leipziger Schule konnte, zumindest in Deutschland, nicht länger als die Berliner Mauer überleben. Doch unter dem Einfluss der Leipziger Schule sind in folgenden Jahren ähnliche Ansätze auch in Amerika und England vorzufinden. Albrecht Neubert zum Beispiel förderte später textlinguistische Übersetzungsforschungen in den USA, wo er sich dank eines Austauschprogramms befand. Auch Christina Schäffner, die der Nachwuchsgeneration der Leipziger Schule angehört, arbeitete später in den USA im Bereich der Übersetzung und der funktionalen Textlinguistik (vgl. Pym 2010: 1-7). Seit 1992 unterrichtet Schäffner an der Aston University in Birmingham u.a. auf dem Gebiet der Übersetzungswissenschaft.

Diese Zeit ist sowohl für Europa als auch für Amerika noch die Nachkriegszeit und geprägt vom Bemühen, internationale Beziehungen wiederherzustellen und Fortschritt in vielen Bereichen wie Infrastruktur, Wirtschaft, Industrie, Architektur und Wissenschaft aufzubauen.

Das Paradigma in diesem modernen Zeitalter, das bis zum späten 20. Jahrhundert reicht, gründet neben dem Ziel der maschinellen Übersetzung auch auf Sprachstruktur und richtet sich streng nach positivistischen Wissenschaftskriterien. In Ansätzen aus dieser Zeit fällt die Suche nach Regelsystemen, einer Übersetzungsgrammatik (vgl. u.a. Newmark 1991, House 1997) und ebenso die präskriptive Wissenschaftshaltung auf.

Der Begriff Äquivalenz taucht erstmals im Zusammenhang mit der Übersetzung auf und wird ausschließlich auf Struktur- bzw. Wortebene bezogen. Es gilt die Forderung

\footnotetext{
${ }^{4}$ Obwohl man keinen direkten Bezug zwischen der Prager Schule und der Leipziger Schule definieren kann, sind durch die oben genannten historischen Entwicklungen die Einflüsse der Prager Schule auf die nächsten Generationen der Übersetzungswissenschaftler(innen) offensichtlich. Diese kommunistischen Regime legten großen Wert auf das Übersetzen: Erstens, weil sie die nationale Sprache entwickelte und zweitens, weil sie ein Mittel zur Ausbreitung ihrer Ideologie darstellte (vgl. dazu auch Pym 2010).
} 
ein Wort für ein Wort, da erste Dateninventare für die maschinelle Übersetzung hergestellt werden.

Der Terminus Übersetzung wird in dieser Periode als Zuordnung von Zeichen und Kodwechsel verstanden, während der Terminus Übersetzer(in) in den betreffenden Ansätzen kaum vorzufinden ist. Der/ die Übersetzer(in), den/ die die Maschine ja ersetzen soll, wird hier als „sprachmittlerisches System“ (Koller 2000: 131f) wahrgenommen, denn der Glaube an die ,[...] Fully Automatic High Quality Translation [...]“(Stolze 2011: 49) ist stark. Da hier die Kriterien der modernen Wissenschaftlichkeit wie zum Beispiel Objektivierbarkeit und Allgemeingültigkeit ausschlaggebend sind, wird der/die Übersetzer(in) als Subjekt in die Übersetzungsforschungen gar nicht erst mit aufgenommen.

So auch in den Ansätzen von Vinay / Darbelnet (1958), Kade (1963), Catford (1965), Malblanc (1968), Reiß (1971), Jakobson (1981), Newmark (1988), Koller (1992) und House (1997) sind die Termini Äquivalenz, Übersetzung und Übersetzer(in) mit den oben genannten Begriffsinhalten vorzufinden.

\section{Unzulänglichkeit der Strukturorientiertheit}

Die zweite Phase, die sich noch immer im modernen Zeitalter bewegt, ist der Übergang von hauptsächlich strukturalistischen Ansätzen zu sogenannten funktionalistischen Ansätzen im späten 20. bis hin zum frühen 21. Jahrhundert. Sie beinhaltet übersetzungswissenschaftliche Forschungsinhalte, die eigentlich auf die neue Generation der Prager und Leipziger Strömung gründen (vgl. Pym 2010: 7f). Neben den Linguisten und Literaturwissenschaftlern arbeiten währenddessen auch Übersetzer(innen) und Dolmetscher(innen) an theoretischen Ansätzen zur Beschreibung und Definition von Begriffen der Äquivalenz, Übersetzung und Übersetzer(in).

Im Laufe der Nachkriegszeit entsteht ein Bedarf an qualifizierten Übersetzer(inne)n und Dolmetscher(inne)n, insbesondere im Rahmen von internationalen Beziehungen in Bereichen wie Wirtschaft, Recht und Politik. Infolge dieses Bedarfs einerseits und der Ankündigung der Notwendigkeit einer Übersetzungswissenschaft als eigenständige Disziplin andererseits, wurden innerhalb dieser Zeitspanne Translationsstudiengänge und universitäre Lehrstühle gegründet, die u.a. zur tatsächlichen Entfaltung der Übersetzungswissenschaft beigetragen haben. Es handelt sich nun nicht mehr nur um Sprachwissenschaftler(innen), sondern auch um Übersetzungswissenschaftler(innen), die in die Disziplin hineingewachsen sind.

Das übersetzungswissenschaftliche Paradigma dieser historischen Periode ist geprägt von der Auffassung, dass ausschließlich strukturorientierte Forschungen für die Definition der Übersetzung unzulänglich sind. Das neue Paradigma baut auf die Deskription auf (vgl. insbesondere Even-Zohar 1990, Toury 1995). Theoretische Ansätze, die in dieser Phase entwickelt werden, streben in erster Linie eine Formulierung von Normen an. Es geht nicht um die Beschreibung von Regeln, die Anweisungen darüber beinhalten, wie eine gute Übersetzung sein soll, sondern um eine Beschreibung, 
die beinhaltet, was eine Übersetzung -unabhängig von ihrer Qualität- überhaupt ist oder sein kann. Eine präskriptive Regelsuche wird zwar allmählich aufgegeben und eine deskriptive Neigung ist wahrzunehmen, jedoch ist eine stark positivistische und strukturorientierte Forschungshaltung immer noch $\mathrm{zu}$ beobachten. Das deskriptive Paradigma scheint sich nämlich nicht von der theoretisierenden Haltung loslösen zu können, was die Quantität der übersetzungstheoretischen Ansätze insbesondere in dieser Periode bestätigt (vgl. Pym 2010: 2f). Als ein Grund dafür wird der anhaltende Einfluss der Prager und Leipziger Schule auch auf diese historische Phase der Übersetzungswissenschaft genannt.

Der aus Israel stammende Itamar Even-Zohar studiert in Kopenhagen und interessiert sich insbesondere für die wissenschaftliche Beschreibung jüdischer Literatur, weshalb er die Ansätze von Jakobson, Tynyanov und Eikhenbaum intensiv rezipiert. Auch sein Zusammenkommen mit Anton Popovič deutet auf sein Interesse am literarischen Strukturalismus. Ausgehend von der heterogenen demographischen Struktur Israels entwickelt er in diesem Zusammenhang die Polysystem Theory (1978, 1990), in der er Kulturen als heterogene und komplexe Systeme mit Subsystemen beschreibend auch übersetzte Literatur als ein Subsystem von Kulturen definiert (vgl. ebd.: 7f; Pym 2014: 62f).

Eine weitere, auch Niederländische Schule genannte, deskriptive Strömung der Übersetzungsforschungen entwickelt sich anfangs der 70er Jahre in den Niederlanden und in Belgien. James S Holmes, José Lambert, Raymond van den Broeck, André Lefevere und Theo Hermans gehören zu dieser Strömung. Wie bekannt, sind auch die Niederlande geprägt durch ihre heterogene demographische Struktur, weshalb das literarische Übersetzen auch hier eine größere Bedeutung innehat. Außer Holmes, der später die Feldtheorie des Übersetzens entwickeln sollte, konzentrierte sich die Niederländische Schule vorwiegend auf Übersetzungen in Bezug auf vergleichende Literaturwissenschaft (vgl. Stolze 2011: 144). Verbunden mit persönlichen Kontakten zu Anton Popovič sind strukturalistische Ursprünge in dieser Schule deutlich zu erkennen (vgl. Pym 2010: 7f). Mit dem Umzug von Holmes nach London und Hermans nach Amerika überschreitet die Perspektive der Niederländischen Schule seine eigenen geographischen Grenzen. Auch die Übersetzungswissenschaftlerin Susan Bassnett befindet sich in England und arbeitet im gleichen Wissenschaftsbereich. Diese Schule wird später The Manipulation School genannt (vgl. Stolze 2011: 144f). Wie aus dem Namen zu entnehmen ist, handelt es sich neben Kultur, Literatur und Übersetzung auch um Manipulation, wobei ihre Ähnlichkeit zu den Ansichten der Prager Schule zu übersetzter Literatur ganz offensichtlich ist.

Der Terminus Äquivalenz wird innerhalb dieser Periode gefordert und auf der Ebene von Funktion, Ziel oder Bedeutung definiert. Er soll nicht mehr ausschließlich im Dienste der Linguistik stehen, sondern soll zu einem Merkmal aller Übersetzungen werden. Schließlich sollen Übersetzungen gegenwärtig nicht mehr als Zuordnung von Sprachstrukturen, sondern unabhängig von ihrer linguistischen oder ästhetischen Qualität als Texte wahrgenommen (vgl. Toury 1980: 63-70; Hermans 1985: 10f). 
Der Terminus Übersetzung wird somit als Produkt (Text), als Prozess oder professionelle Handlung im Sinne von einer Bedeutungsübertragung nach dem Ziel des Übersetzungsauftrages definiert. Dem Terminus Übersetzer(in) wird der Begriffsinhalt Experte zugeordnet, der zwar über eine Entscheidungskraft verfügt, aber stets objektiv und transparent bleiben muss. Seine Entscheidungen werden von der Übersetzungssituation bestimmt.

Die deskriptive Forschungsrichtung mit theoretisierendem Inhalt und die Begriffe Äquivalenz, Übersetzung und Übersetzer(in) mit den oben genannten Begriffsinhalten sind in den Ansätzen von Reiß / Vermeer (1984), Hermans (1985), Holz-Mänttäri (1986), Snell-Hornby (1988), Holmes (1988), Even-Zohar (1990), Bassnett (1991), Nord (1991), Lefevere (1992), Toury (1995), und vielen anderen Übersetzungswissenschaftlern dieser paradigmatischen Zeitspanne vorzufinden.

\section{Sociologic Turn}

Die dritte historische Phase der in dieser Untersuchung genannten Phasen der Übersetzungsforschung, die ungefähr gegen Ende des 20. Jahrhunderts bis Anfang des 21. Jahrhunderts datiert werden kann, besteht größtenteils aus Ansätzen von transnationalen Kulturtheoretikern, Soziologen, Ethnographen, Psychoanalytikern und Übersetzungswissenschaftlern, die vor allem aus Amerika und Europa stammen.

Die übersetzungswissenschaftlichen Ansätze in der dritten Phase gründen hauptsächlich auf postmodernere Theorien, die synchron $\mathrm{zu}$ den modernen funktionalistischen Ansätzen entwickelt werden. Diese paradigmatische Phase der Übersetzungswissenschaft unterscheidet sich von den anderen dadurch, dass sie die wissenschaftliche Haltung und die wissenschaftlichen Kriterien der Moderne kritisiert. In den übersetzungswissenschaftlichen Ansätzen dieser Zeit wird betont, dass ausschließlich objektive und theoretisierte Erkenntnisse den/ die Sprachnutzer(in), den geschichtlichen Kontext und die Kultur im Sinne von Subjekt/ Ort/ Zeit ausschließt und somit die Realität verfehlend, die Kluft zwischen Theorie und Praxis nur noch weiter aufgerissen wird. Dabei stützt sich das wissenschaftliche Paradigma dieser Zeitspanne besonders auf ideologische Machtstrukturen (vgl. Stolze 2011: 203). In diesem Sinne wird auf die variablen Faktoren sowohl im Sprachgebrauch als auch in der Übersetzung verwiesen und das Übersetzen wird dabei nicht mehr aus den Perspektiven der Linguistik oder der Literatur, sondern aus den Perspektiven der postmodernen Soziologie, des Postkolonialismus, der kulturellen Hybridität, des Rassismus, der Geschlechterdiskriminierung, der Ideologie, der Zensur oder der Migration betrachtet (vgl. Pym 2014: 138; Stolze 2011: 203f). Interessant ist die Ähnlichkeit mit dem Ausgangspunkt der Prager Schule, in der es ursprünglich ebenfalls um die Veränderung der nationalen Literatur mit Hilfe der Translation ging, auch wenn entscheidende Unterschiede in geschichtlichem Kontext und der Begriffsdefinitionen vorliegen. Ein weiteres Merkmal dieses Paradigmas ist der Ausgangspunkt, dass die Übersetzung nicht mehr mit der internationalen Mobilität von Texten in Verbindung gesetzt wird, sondern mit der internationalen Mobilität von Menschen ihren Ursprung findet (vgl. ebd.: 142). 
In dieser Zeitspanne interessieren sich die Übersetzungsforscher(innen) überwiegend weder für die Frage, wie eine gute Übersetzung aussehen muss, noch dafür, was eine Übersetzung ist. Das Interesse hat sich auf die Fragestellung verlagert, was überhaupt übersetzt wird, warum gerade diese Übersetzungen vorgenommen wurden, wer die Textauswahl getroffen hat, welche Verlagspolitik verfolgt wird und welche Zwänge damit in Kraft treten (vgl. Stolze 2011: 203). Das Augenmerk richtet sich somit auf eine neue Strömung unter dem Namen cultural turn (Bassnett / Lefevere 1998), die die Loslösung des Übersetzungskonzepts vom rein linguistisch-textlichen Paradigma in Richtung Kultur und Ideologie vorsieht.

Eine ausgesprochen neue Perspektive bietet diesbezüglich die ,postmoderne übersetzerische Ästhetik" von Edwin Gentzler. Er verwendet den Begriff der Übersetzung mit dem „Kannibalismus“ und vertritt dabei die Meinung, dass der/ die Übersetzer(in) das im Original Vorgefundene „,verschlingen“ würde, und dass die Übersetzung das Original übertreffen müsse (Gentzler 1993: 192f). In diesem Kontext wird dem/der Übersetzer(in) eine wichtige soziale und ideologische Rolle zugeschrieben. Die Äquivalenzbeziehung zwischen dem Ausgangs- und Zieltext spielt keine große Rolle mehr, wichtiger ist die Transtextualisierung, d. h. die Beziehung des Übersetzungstextes zu anderen Texten in der Zielkultur.

In dieser dritten paradigmatischen Phase findet die Übersetzungsforschung auch einen Bezug zu feministischen Aspekten. Unter dem Namen écriture feminine kritisiert eine Gruppe von Autorinnen in ihren Werken mittels Schreibstil die männlich dominierte Schreib- und Denktradition, weil ihr die Ursache der Geschlechterdiskriminierung zugrunde liegt. $\mathrm{Zu}$ ihrem Stil gehört die Verachtung von strukturalen Sprachnormen, u. a. auch dem diskriminierenden Artikelgebrauch. Die deutsch-kanadische Literaturkritikerin Luise von Flotow (1997) macht diesen Ansatz für das Übersetzen fruchtbar. Im Jahre 1999 beginnt Michaela Wolf in Österreich (Graz) mit einem Projekt namens Integration von Theorie und Praxis feministischer Translation, in dem eine umfassende Dokumentation über feministische Translation in Forschung, Lehre und Praxis im deutschen Sprachraum erarbeitet wird (vgl. Stolze 2011: 208f).

Die Kritik an feministischen Ansätzen unterstreicht, dass sie in einem eurozentrischen Diskurs verbleiben, obwohl Frauen aus Ländern der sogenannten Dritten Welt der Diskriminierung weitaus mehr ausgesetzt seien und dadurch der Feminismus den Rassismus degradiere. „Der emanzipatorische Charakter von Übersetzungen sollte weitere Unterdrückungsformen einschließen“ (ebd.: 213).

Daraufhin verlagert sich das Interesse auch auf den Rassismus, den Postkolonialismus und die Kultur bzw. die Subkultur und die Hybridität. In diesem tritt die Forderung nach einer ideologischen Initiative des (der) Übersetzers(-in) auf. Minderheitskulturen sollen sich von kolonialistischen Kulturen und Normen lösen und Übersetzungen wiederum sollen sich von Normen des Originals lösen. Die ethische Aufgabe des (der) Übersetzers(-in) liege darin, die einheimische Tradition zu bewahren und die Übersetzung als permanenten Bedeutungswandel des Originals in den Vordergrund zu rücken (vgl. ebd.: 213-216). 
Die aus Indien stammende Literatin, Übersetzerin und postkoloniale Theoretikerin Gayatri Chakravorty Spivak (2007) hat sich in Amerika mit Sprachgebrauch und Geschlechterdiskurs auseinandergesetzt, während ihre theoretischen Ansätze auch Definitionen zum Übersetzen umfassen. Nach Spivak ist das Übersetzen eine subjektive und kulturelle Umwandlung (vgl. Spivak 2007: 261). Spivak ist stark geprägt von Derridas Dekonstruktion und sieht das Übersetzen als eine Art Wiederbelebung des Originals.

Der Terminus Übersetzung wird unter diesem Paradigma als Mittel zum Zweck ideologischer Intentionen definiert. Sie gilt in dieser historischen Phase als ,[...] ein Faktor bei der Konstruktion von Wissen und Kultur [...]" (Stolze 2011: 203). Obwohl es um denselben Terminus wie in der ersten und zweiten paradigmatischen Phase der Übersetzungswissenschaft geht, handelt es sich nicht wirklich um Übersetzung als Text oder Inhaltsübertragung, sondern um Übersetzung als ein Faktor in der Kommunikation zwischen größeren oder kleineren kulturellen Gemeinschaften (vgl. Pym 2014: 138). Homi K. Bhabha zum Beispiel definiert das Originalwerk The Satanic Verses des transnationalen Schriftstellers Salman Rushdie als Translation, als Veränderung kultureller und religiöser Komponenten. Seines Erachtens gibt es nichts Unübersetzbares oder Mehrdeutiges, es gibt keine Iterabilität, sondern nur einen Widerstand, sich dem Original zu integrieren (vgl. ebd.: 140f). Bhabha (2004) konzentriert sich in seiner Arbeit How Newness Enters the World: Postmodern Space, Postcolonial Time and the Trials of Cultural Translation nicht mehr auf den literarischen Text, sondern den/ die Schriftsteller(in) als Person, während es sich bei Übersetzung nicht mehr um Mehrsprachigkeit, sondern um nur eine Sprache (Englisch) handelt. Übersetzung ist aus dieser Perspektive gesehen nicht die Übertragung eines ausgangssprachlichen Textes in einen zielsprachlichen Text, sondern allein die Übertragung von kulturellen und soziologischen Inhalten und Gedanken in Originaltexte.

Aus den obigen Darstellungen zeigt sich, dass die Übersetzung nicht als strukturales Produkt, sondern als kultureller Prozess wahrgenommen wird. Aufgrund dieser Prozessorientiertheit ist die Forderung nach Äquivalenz nicht mehr auf der sprachstrukturellen Ebene aufzufinden. Falls es überhaupt noch um den Begriff der Äquivalenz geht, dann sind umfassendere und flexiblere Definitionen in Verbindung mit Begriffen wie Übersetzer(in), Kompetenz, Kontext und Situation zu in Betracht zu ziehen (vgl. u.a. Nord 2017). Es ist zu beobachten, dass innerhalb dieses Zeitraums außersprachliche Faktoren in der übersetzungswissenschaftlichen Forschung mehr Aufmerksamkeit erhalten als sprachstrukturelle Faktoren.

Der Begriff Übersetzer(in) wird weder aus maschineller noch aus objektiver Perspektive definiert. Unter diesem Paradigma kann der/ die Übersetzer(in) nicht mehr mit Adjektiven wie objektiv oder transparent definiert werden, sondern er/ sie darf und soll sogar als ein ideologische(r) Aktivist(in) agieren, denn die Übersetzung wird als eine fundamental ethnozentrische Kommunikation unter Ungleichen wahrgenommen, wogegen der/ die Übersetzer(in) sich wehren soll (vgl. Venuti 1995: 93). Hier werden Begriffe wie Kultur, Kritik, Ideologie und Macht mit in den wissenschaftlichen Ansätzen integriert und werden nicht mehr wie in der ersten Phase der Moderne aus den 
Wissenschaften ausgeschlossen. In Forschungen innerhalb dieser Zeitspanne fällt erstmals eine wirkliche Subjektorientiertheit auf, in dem der/ die Übersetzer(in) als interpretierender Leser wahrgenommen wird, für den eine ideologische Transparenz unmöglich scheint.

Die in dieser Arbeit angegebene Tabelle 1 zeigt die Definitionen der drei Begriffe Äquivalenz, Übersetzung und Übersetzer(in) gemäß den oben zusammengefassten drei historischen und paradigmatischen Phasen, die in der Tabelle unter den Spalten Phase 1, Phase 2 und Phase 3 dargestellt sind und jeweils andere Paradigmen beinhalten. In Tabelle 1 ist unter der Zeile Subjekt die jeweilige Forschungsgesellschaft zu verstehen, die unter dem derzeit herrschenden Paradigma federführende wissenschaftliche Ansätze entwickelte. Die Zeile Ort beinhaltet Angaben darüber, in welchem geographischen Raum derzeit relevante Forschungen zur Übersetzung überwiegend stattgefunden haben, während die Zeile Zeit die Zeitspanne des betreffenden Paradigmas angibt.

\begin{tabular}{|c|c|c|c|}
\hline \multicolumn{4}{|c|}{ Historischer Wandel der Definitionen von übersetzungswissenschaftlichen Termini } \\
\hline & I. Phase & II. Phase & III. Phase \\
\hline$\Xi$ & $\begin{array}{l}\text { Mitteleuropa, } \\
\text { Europa, Amerika }\end{array}$ & $\begin{array}{l}\text { Mitteleuropa, Europa, } \\
\text { Niederlande, Amerika, } \\
\text { England, Mittelosten; } \\
\text { Später: Verbreitung auf } \\
\text { der ganzen Welt }\end{array}$ & Amerika, England, Europa \\
\hline$\frac{2}{3}$ & $\begin{array}{l}\text { Literaten, } \\
\text { Linguisten: } \\
\text { Prager Schule, } \\
\text { Leipziger Schule; } \\
\text { Popovič, } \\
\text { Mukařovský, } \\
\text { Jakobson, } \\
\text { Trubetzkoi, } \\
\text { Becker, Jäger, } \\
\text { Neubert, Kade, } \\
\text { Newmark, Catford, } \\
\text { Vinay / Darbelnet, } \\
\text {... }\end{array}$ & $\begin{array}{l}\text { Reflexionen der Prager } \\
\text { und Leipziger Schule, } \\
\text { neue Generation; } \\
\text { Linguisten; } \\
\text { Literaten; } \\
\text { Übersetzer/Dolmetscher; } \\
\text { Ü-wissenschaftler; } \\
\text { Even-Zohar, Toury, } \\
\text { Snell-Hornby, Holmes, } \\
\text { Holz-Mänttäri, } \\
\text { Lefevere, Bassnett, } \\
\text { Nord, Reiß / Vermeer, } \\
\text { Hermans u.v.a. }\end{array}$ & $\begin{array}{l}\text { Ü-wissenschaftler; } \\
\text { Kulturtheoretiker; } \\
\text { Ethnographen; } \\
\text { Soziologen; } \\
\text { Psychoanalytiker; } \\
\text { Bhabha, Venuti, Spivak, } \\
\text { Tymoczko, von Flotow, } \\
\text { Douglas... }\end{array}$ \\
\hline$\stackrel{\widetilde{\Xi}}{\sim}$ & $\begin{array}{l}\text { Moderne: } \\
\text { ab Mitte des 20. Jh. } \\
\text { - spätes 20. Jh. }\end{array}$ & $\begin{array}{l}\text { Moderne: } \\
\text { spätes 20. Jh. - frühes } \\
\text { 21. Jh. }\end{array}$ & $\begin{array}{l}\text { Postmoderne: } \\
\text { 21. Jh. }\end{array}$ \\
\hline
\end{tabular}




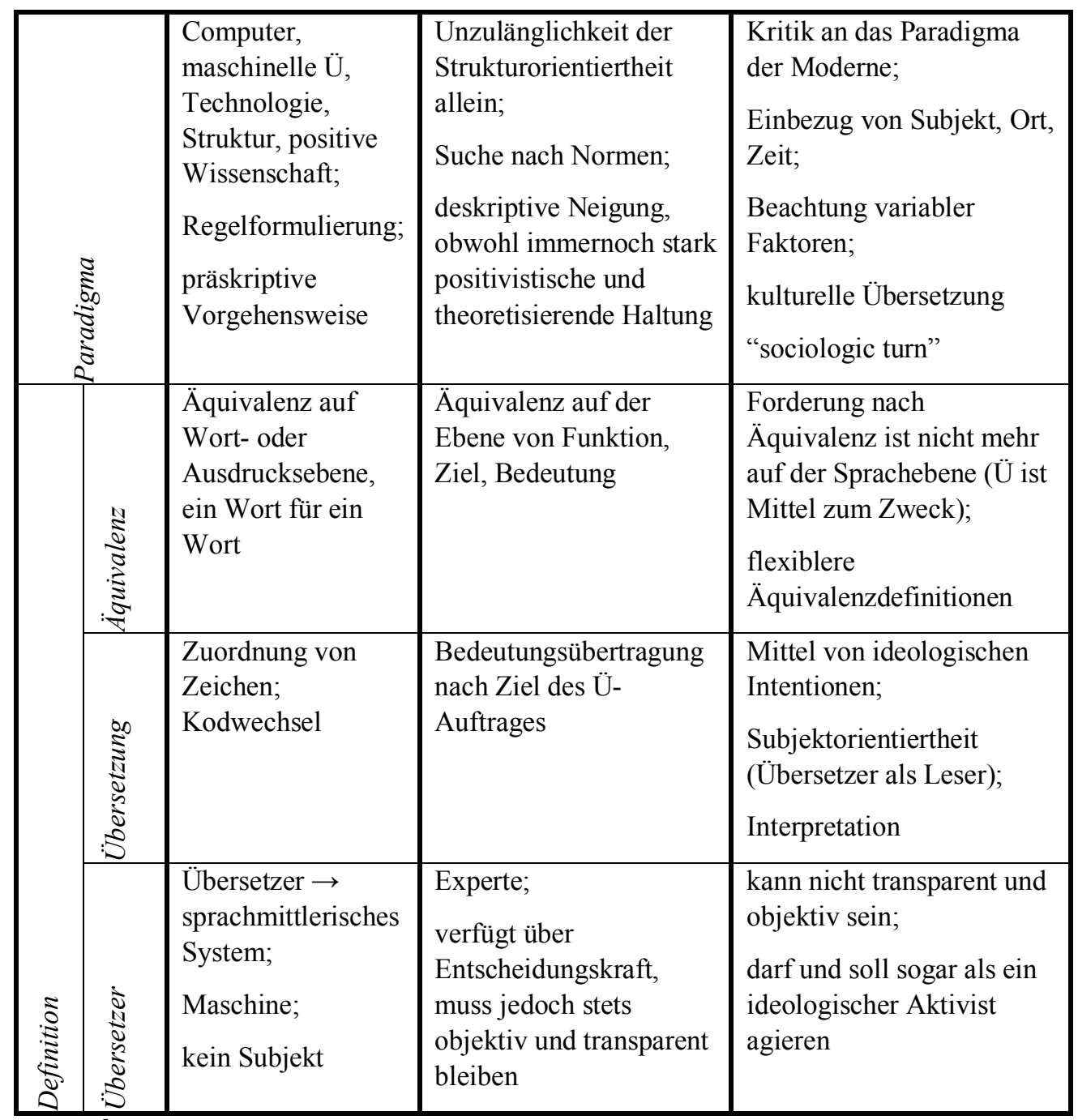

Tab. $1^{5}$ : Historischer Wandel der Definitionen von übersetzungswissenschaftichen Termini

\section{Schlussfolgerung}

Historisch und chronologisch gesehen kann man weder aus theoretischer noch aus begrifflicher Hinsicht von einer Unübersichtlichkeit innerhalb der Übersetzungswissenschaft sprechen. Wie aus der oben dargestellten Diskussion deutlich hervorgeht, erhält die Vielfältigkeit der Terminidefinitionen der Übersetzungswissenschaft einen Sinn, sobald diese Definitionen zusammen mit der historischen Periode bewertet werden. Parallel zum Paradigmenwechsel in der Übersetzungswissenschaft wird auch der Bedeutungsinhalt eines Terminus von Übersetzungsforscher(inne)n neu definiert und begrifflich ,wieder belebt“. Daraus ist zu folgern, dass übersetzungswissenschaftliche Arbeiten nicht kontextlos vorgehen dürfen. Insbesondere die Analyse und Interpretation der Termini in der Übersetzungswissenschaft oder die darauf gründende Forderung nach allgemeingültigen theoretischen Erkenntnissen hat u.a. historische Vorgänge zu beachten, wenn es nicht

\footnotetext{
${ }^{5}$ Seitens der Verfasserin für diese Untersuchung angefertigte Tabelle
} 
darum gehen soll, die Kluft zwischen der Theorie und Praxis von Übersetzung noch weiter aufzureißen.

Interpretiert aus der dekonstruktivistischen Perspektive mit den Begriffen Iterabilität und Dissemination, kommt deutlich zum Vorschein, dass es sich im Rahmen der Definitionen übersetzungswissenschaftlicher Termini nicht um eine Unübersichtlichkeit, sondern um einen historisch bedingten paradigmatischen Wandel handelt. Dieser Wandel bedingt nicht die Ungültigkeit der Forderung der Terminologiearbeit über möglichst genau definierte Termini innerhalb eines Forschungsfeldes. Aus der oben dargestellten historischen Analyse und der daraus erstellten Tabelle 1 wird evident, dass es nur innerhalb einer paradigmatischen Zeitspanne Einigkeit über den begrifflichen Inhalt bestimmter Termini gibt.

Aus den oben ausgeführten theoretischen und historischen Darstellungen ist zu folgern, dass die Universalität von Terminidefinitionen der Übersetzungswissenschaft keine ideale Universalität im philosophischen Sinne, sondern nur eine Universalität im Rahmen eines bestimmten historischen Kontextes beinhalten kann. Aus dieser Perspektive gesehen ist die Erwartung auf eine rein universalistische Definition von übersetzungswissenschaftlichen Termini nicht realistisch und passt auch nicht in die Kriterien der Wissenschaftlichkeit, in denen die Erwartung oder Behauptung von absoluter Richtigkeit bestimmter Erkenntnisse auch keine Gültigkeit hat.

Auf reine Verallgemeinerungen fokussiertes, rein theoretisch orientiertes, rein abstrahierendes und rein strukturorientiertes übersetzungswissenschaftliches Arbeiten neigt zu einer extremen Distanz zur Praxis und kann somit die Funktion und das Ziel der Übersetzungswissenschaft verfehlen. In diesem Zusammenhang scheint es sinnvoller, sowohl die Übersetzungspraxis als auch die Übersetzungswissenschaft verbunden mit variablen Faktoren und historischen Entwicklungen zur Hand zu nehmen.

\section{Literaturverzeichnis}

Arntz, Reiner / Picht, Heribert u.a. (2004): Einführung in die Terminologiearbeit (= Studien zu Sprache und Technik 2). 5., verbesserte Auflage. Hildesheim: Georg Olms.

Atkins, Douglas. G. (1983): Reading Deconstruction. Deconstructive Reading. Kentucky: University Press of Kentucky.

Bassnett, Susan / Lefevere, Andre (Hg.) (1998): Constructing Cultures. Essays on Literary Translation. Clevedon, Philadelphia, Adelaide: Multilingual Matters.

Bleicher, Knut (1992): Das Konzept Integriertes Managment. 2. Auflage. Frankfurt am Main: Camous Verlag.

Bühler, Karl (1965): Sprachtheorie. Die Darstellungsfunktion der Sprache. 2. Auflage. Stuttgart: UTB Verlag.

Catford, John C (1965): A Linguistic Theory of Translation. An Essay on Applied Linguistics. London: Oxford University Press.

De Man, Paul (1979): Shelley Disfigured. In: De Man, Paul / Derrida, Jacques u.a. (Hg.): Deconstruction and Criticism. London/ Henley: Continuum International Publishing Group, 39-73.

Derrida, Jacques (1967): L'écriture et la différance. Paris: Éditions du Seuil. 
Derrida, Jacques (1972): Die Schrift und die Differenz. (Übersetzt von: Rodolphe Gasché.) Tübingen: Suhrkamp Verlag.

Derrida, Jacques (1974): Grammatologie. (Übersetzt von: Rheinberger Hans-Jörg/ Zischler, Hanns) Frankfurt am Main: Suhrkamp Verlag.

Derrida, Jacques (2007): Psyche. Inventions of the Other. Volume I. Stanford/California: Stanford University Press.

DIN 2342 (2011): Begriffe der Terminologielehre. Berlin/Köln: Beuth Publishing. 22.

Gile, Daniel (2009): Basic Concepts and Models for Interpreter and Translator Training. Revised Edition. Amsterdam/Philadelphia: John Benjamins Publishing Company.

Hermans, Theo (1985): Translation Studies and A New Paradigm. In: Hermans, Theo (Hg.): The Manipulation of Literature. Studies in Literary Translation. London, Sydney: Croom Helm, 7-16.

Hoffmann, Lothar (1993): Fachwissen und Fachkommunikation. Zur Dialektik von Systematik und Linearität in den Fachsprachen. In: Bungarten, Theo (Hg.): Fachsprachentheorie. Bd. Nr. 2. Tostedt: Attikon Verlag, 595-617.

House, Juliane (1997): Translation Quality Assessment. A model revisited. Tübingen: Gunter Narr Verlag.

Hörz, Herbert (1999): Wissenschaft als Aufklärung? - Von der Postmoderne zur Neomoderne. In: Sitzungsberichte der Leibniz-Sozietät, Heft 1. Band 28, 5-86.

Jakobson, Roman (1981): Linguistische Aspekte der Übersetzung. In: Wilss, Wolfram (Hg.): Übersetzungswissenschaft. Darmstadt: Klett Verlag, 189-198.

Kade, Otto (1963): Aufgaben der Übersetzungwissenschaft: Zur Frage der Gesetzmäßigkeit im Übersetzungsprozeß. In: Fremdsprachen 7, Nr. 2, 83-94.

Kluge, Friedrich (2002): Etymologisches Wörterbuch der deutschen Sprache. 24., durchgelesene und erweiterte Auflage, bearbeitet von Elmar Seebold. Berlin: de Gruyter Verlag.

Koller, Werner (1992): Einführung in die Übersetzungswissenschaft. 4. völlig neu bearbeitete Auflage, ${ }^{7}$ 2004. Heidelberg und Wiesbaden: A. Francke Verlag UTB.

Koller, Werner (1995): The Concept of Equivalence and the Object of Translation Studies. In: Target 7(2), 191-222.

Koller, Werner (2000): Grundfragen der Übersetzungswissenschaft - Nachtrag zu Leipzig 1970. In: Schmitt, Peter A. (Hg.): Paradigmenwechsel in der Translation. Festschrift für Albrecht Neubert zum 70. Geburtstag. Tübingen: Stauffenburg Festschriften, 121-135.

Kuhn, Thomas (2003): Die Struktur wissenschaftlicher Revolutionen. Frankfurt am Main: Suhrkamp Verlag.

Lévi-Strauss, Claude (1968): Das wilde Denken. Übersetzt von: Hans Naumann. Frankfurt am Main: Suhrkamp Verlag.

Malblanc, Alfred (1968): Stylistique comparée du français et de l'allemand. Essai de reprentation linguistique comparée et étude de traduction. Paris: Didier.

Newmark, Peter (1988): A Textbook of Translation. London / New York: Prentice Hall Publishing.

Pym, Anthony (2010): Exploring Translation Studies. Additional chapter: descriptions - the intellectual background. London/ New York: https://www.researchgate.net/publication/39728790_Exploring_Translation_Theories (Letzter Zugriff: 22.09.2021).

Pym, Anthony (2014): Exploring Translation Theories. Second Edition. London/New York: Routledge. 
Pym, Anthony (2015): The Medieval Postmodern in Translation Studies. In: Fuertes, Alberto / TorresSimon u.a. (Hg.): And Translation Changed the World (and the World Changed Translation). Cambridge: Cambridge Scholars Publishing, 105-122.

Reiß, Katharina (1971): Möglichkeiten und Grenzen der Übersetzungskritik. München: Hueber Verlag.

Schäffner, Christina (2000): Kontinuität und Erneuerung. In: Schmitt, Peter A. (Hg.): Paradigmenwechsel in der Translation: Festschrift für Albert Neubert zum 70. Geburtstag. Tübingen: Stauffenberg Festschriften, 205-216.

Snell-Hornby, Mary (1988): Translation Studies: An Integrated Approach. Amsterdam, Philadelphia: John Benjamins Publishing.

Sonneveld, Helmi / Loening, Kurt (1993): Terminology: Application in Interdisciplinary Communication. Amsterdam, Philadelphia: John Benjamins Publishing.

Stachowitz, Rolf (1973): Voraussetzungen für Maschinelle Übersetzung: Probleme, Lösungen, Aussichten. Frankfurt am Main: Athenäum Verlag.

Stolze, Radegundis (2011): Übersetzungstheorien. Eine Einführung. Tübingen: Gunter Narr Verlag.

Toury, Gideon (1980): In Search of a Theory of Translation. Tel Aviv: Porter Institute for Poetic and Semiotics.

Toury, Gideon (1995): Descriptive Translation Studies and Beyond. Amsterdam-Philadelphia: John Benjamins Publishing.

Venuti, Lawrence (1995): The Translator's Invisibility. A History of Translation. London und New York: Routledge.

Vinay, Jean-Paul / Darbelnet, Jean (1958): Stylistique comparée du français et de l'anglais. Methode de traduction. Paris: Didier.

Wahrig, Gerhard (1997): Deutsches Wörterbuch. Neu herausgegeben von Dr. Renate Wahrig-Burfeind. Mit einem Lexikon der deutschen Sprachlehre. Gütersloh: Bertelsmann Lexikon Verlag.

Wengenroth, Ulrich (2012): Zur Einführung: Die reflexive Modernisierung des Wissens. In: Wengenroth, Ulrich (Hg.): Grenzen des Wissens - Wissen um Grenzen. Weilerswist: Velbrueck GmbH, 7-22.

Wilss, Wolfram (1977): Übersetzungswissenschaft. Probleme und Methoden. Stuttgart: Klett Verlag.

Zima, Václav Peter (1994): Die Dekonstruktion. Einführung und Kritik. Tübingen / Basel: A. Francke Verlag, UTB für Wissenschaft. 